\title{
Juventud indígena en el Totonacapan Veracruzano
}

\author{
Ariel García Martínez
}

Resumen: El objetivo de este artículo es dar a conocer la especificidad de la juventud rural e indígena de Totonacapan, su incorporación a la sociedad adulta y su interacción con la sociedad indígena y no indígena, a través de un comparativo que toma como punto de partida la integración de los poblados pertenecientes a la región conocida como sierra de Papantla, en el norte del Estado de Veracruz con el resto de la sociedad nacional. El trabajo intenta reconstruirel surgimiento de los "jóvenes indígenas" totonacos como producto de la interacción de su propia cultura y la cultura nacional en las últimas cuatro décadas. Estos fenómenos suscitaron diversos cambios en los estilos de vida y la cultura de las comunidades, entre las que se destaca el surgimiento de nuevas identidades.

Palabras clave: Juventud rural, Juventud indígena, nuevas identidades.

Enviado a dictamen: 19 de enero de 2012

Aprobación: 30 de mayo de 2012

Revisiones: 1

Mtro. Ariel García Martínez, Maestro en Antropología Social por el Centro de Investigaciones y Estudios Superiores en Antropología Social (CIESAS-DF). Actualmente colabora en el Proyecto "Asociaciones y Redes sociales en la Zona Metropolitana de Xalapa, Veracruz", Temas de especialización: Juventud Indígena y Ruralen Veracruz. Correo electrónico: aire68@gmail.com.
Abstract: The purpose of this paper is to know the specificity of the rural and indigenous youth in the region of Totonacapan, their incorporation into adult society and their interaction with indigenous and non-indigenous society, through a comparative which takes as a starting point the integration of the villages belonging the region known as Sierra Papantla, in the North of the State of Veracruz with the rest of the national society. The work attempts to reconstruct the emergence of "indigenous youth" among the Totonac people as a result of the interaction of their own culture and national culture in the last four decades. These phenomena raised various changes in the lifestyles and culture of the communities including the emergence of new identities.

Keywords: Rural youth, indigenous youth, new identities.

$\mathrm{E}$ 1 presente artículo ${ }^{1}$ tiene como propósito ofrecer una visión panorámica sobre los aspectos de la juventud rural de Totonacapan, particularmente de la juventud indígena ubicada en los municipios serranos del norte del estado mexicano de Veracruz (donde se incluyen los municipios de Coyutla, Espinal, Mecatlán y Coahuitlán, entre otros), a partir de un enfoque que considera a la juventud "como una construcción sociocultural, históricamente definida” (Valenzuela, 1997: 13). 
Un antecedente para entender la emergencia de los estudios sobre la juventud nos remite a recordar que en México, después de las históricas revueltas estudiantiles de los años sesenta y principios de los setenta, la categoría "estudiante" definió por metonimia la esencia de lo que significaba ser "joven". Posteriormente, la crisis económica en la década del ochenta colocó a los jóvenes pobres como aquellos que definen el concepto. La cultura de las "bandas" se presentó y representó como una cultura de resistencia al modelo de la sociedad adulta, cuyas principales demandas provenían de una realidad marcada por el desempleo y la represión. Las investigaciones antropológicas se orientaron entonces al estudio de estos jóvenes proletarios, habitantes de los cinturones de miseria de las grandes ciudades. Estos espacios marginales urbanos eran el territorio donde se generaban nuevas maneras de ser joven, donde los "chavos banda" construían un imaginario y una interpretación de su realidad rechazados por una sociedad que creía ser moderna (García, 2009).

Poco a poco, estos enfoques se han ampliado a través de las aportaciones de especialistas que han fincado un nuevo corpus de conocimientos sobre la materia. Los estudios de género y de perspectiva étnica han enriquecido estos saberes sobre los complejos sujetos sociales a los que, por comodidad expositiva, denominaré "juventudes". Las contribuciones de Rosana Reguillo Cruz (2010), José Manuel Valenzuela (2009), Maritza Urteaga Castro-Pozo (2010) y Maya Pérez Ruiz (2008), entre otros autores, han abonado el terreno para que el abordaje del estudio de las juventudes indígenas y rurales se desarrolle sobre bases firmes, marcando una trayectoria investigativa desde principios de los años noventa en el extinto siglo pasado, hasta el momento presente.

El estudio de lo "juvenil", según Rossana Reguillo, tiene mucho que ver con los "complejos procesos sociohistóricos que catapultaron a la escena pública a los jóvenes como actores sociales emergentes" (Reguillo, 2010: 9). Por lo tanto, el desarrollo de un estudio y teoría de la juventud está enlazada con la visibilidad del fenómeno, sobre todo en sociedades urbanas e industrializadas. Esto también explica la abundante literatura que se ha escrito sobre las juventudes occidentales, por lo menos a partir de las primeras décadas del siglo XX, desde una perspectiva sociológica y psicológica.

A diferencia del estudio de las juventudes urbanas, los estudios sobre su contraparte indígena se han desarrollado de manera tardía. Maritza Urteaga CastroPozo certeramente destaca la importancia que ha tenido el tema de las poblaciones originarias en la antropología mexicana y lo poco que se ha dicho sobre la juventud en estas sociedades. Establece que son varias las razones por las que no se han realizado dichos estudios, entre las cuales destaca, de manera central, la reciente existencia del fenómeno entendido como:

Un periodo etario que pudiera ser reconocido por la sociedad étnica bajo estudio como diferenciado de la infancia y la adultez. En los últimos años se han registrado cambios en la cantidad y calidad de los estudios y aproximaciones al tema de los jóvenes indígenas. Aun siendo muy pocos, demuestran el creciente interés de jóvenes antropólogos en un nuevo fenómeno: la emergencia de algo que puede denominarse periodo juvenil entre la población étnica que habita tanto en los pueblos como en las ciudades (Urteaga Castro-Pozo, 2010: 35)

En este artículo se estudian las peculariadades de esta emergencia de lo "juvenil étnico" en una región indígena. Estas peculiaridades tienen que ver con los procesos históricos que intervienen en la construcción de una categoría como "juventud" en sociedades donde previamente no existía (Urteaga Castro-Pozo, 2008). Lo anterior nos conduce a examinar una serie de fenómenos adyacentes como la interacción de las sociedades indígenas con novedosos agentes externos, las fuerzas de la política, la tecnología y el mercado 
en plena era de la globalización. De manera creciente estas comunidades enfrentan problemas de asimetría, resistencia y desigualdad de género al interior de sus propias sociedades, a los cuales habría que añadir problemas añejos como la explotación, la pobreza y la discriminación (Pérez Ruiz, 2008). Es por ello que se hace necesario entender cómo se gestan estas nuevas identidades en el marco de las grandes transformaciones globales.

Para el estudio de este caso particular, he decidido considerar a la juventud indígena como un periodo vital reciente entre los totonacos, periodo marcado en términos biológicos con la entrada a la pubertad y, en términos sociales, con la interrupción del tránsito del estado infantil al adulto de acuerdo a la ruta establecida por la propia cultura (García, 2009). Este periodo se caracteriza por la generación de un nuevo estatus al interior de la sociedad y el surgimiento de procesos identitarios de autopercepción y heteropercepción. Defiendo, en su generalidad, que las sociedades consideradas como tradicionales históricamente han establecido una relación conflictiva y desigual con la sociedad nacional, ${ }^{2}$ a la par que en situaciones de contacto dinámico en su interior se van gestando nuevas identidades que se ensamblan o articulan en un contexto de cambio global.

De esta manera, propongo que se puede hablar de una juventud indígena en situaciones donde la sociedad nacional ha alterado sustancialmente el estilo y forma de vida indígena a partir de factores específicos como la educación a través del sistema escolar; la comunicación a través de medios masivos como la televisión, la radio, internet y la telefonía celular); la economía, por la articulación de la producción regional (café y productos agrícolas) al gran mercado nacional y mundial, además de los procesos migratorios derivados. Estos factores van a impactar en la organización social de los pueblos donde las estructuras tradicionales van a generar nuevos espacios de identidad a lo que he denominado "juventud indígena". Esta juventud es heredera de todo el acervo cultural de sus pueblos y al mismo tiempo selecciona aquellos elementos que le permiten dialogar con la sociedad nacional, readecuando y cotejando los papeles que como sociedad indígena les ha asignado en un marco poscolonial de referencia. Esta readecuación genera un estado de tensión en los roles asignados tradicionalmente a los hombres y mujeres al interior de la sociedad tradicional y, al mismo tiempo, significan un replanteamiento de la sociedad mestiza local, a la que ellos mismos identifican como la "gente de razón" o luhuanes.

Como advertencia, debo señalar que no considero a la sociedad indígena totonaca como estática e inalterable, antes bien, creo que los cambios culturales son un recurso de sobrevivencia ante las presiones externas que se han venido presentando desde hace cuatro décadas, cuando se iniciaron los trabajos para mejorar los caminos que comunican esta región con ciudades más cercanas como Poza Rica y Papantla (García, 2009).

\section{Ubicación geográfica}

El Totonacapan veracruzano contemporáneo es una región comprendida entre la cuenca de los ríos Cazones al norte y Tecolutla al sur, al este el Golfo de México y al oeste la Sierra Norte de Puebla (Velázquez, 1996).

Una región históricamente integrada por condicionamientos económicos y políticos. Valga decir que durante todo el siglo XIX el Totonacapan sufrió una serie de transformaciones:

que estuvieron vertebradas por el eje de la modernización y el cambio social, proceso que encontró su punto culminante durante los últimos años del régimen porfirista. Como consecuencia de ello se modificó la estructura de la sociedad colonial, acelerándose la aculturación de la sociedad totonaca... los cambios que ocurrieron en las fronteras étnicas del Totonacapan a lo largo del siglo XIX muestran 
que éstas no fueron estáticas sino que respondieron a dinámicas históricas y configuraciones regionales que les dieron sentido y las definieron como tales (Chenaut, 1995: 225).

Los cambios se vieron acelerados a partir del primer tercio del siglo XX con el desarrollo de la industria petrolera que reconfiguró el ordenamiento económico de la región, a la par que se generaban nuevos centros urbanos como Poza Rica. Por otra vía, en el ámbito rural, se fortalecían núcleos de población como Coyutla, espacio central de esta investigación, que durante el último cuarto del siglo XX se constituye en la cabecera de una porción serrana del Totonacapan Veracruzano al hallarse en una obligada vía de acceso a diversas comunidades del propio municipio y a municipios vecinos como Jopala, Mecatlán, Filomeno Mata y Coahuitlán.

A pesar de las transformaciones que ocurrieron durante el siglo XIX y buena parte del XX, la sociedad totonaca pudo reproducir al nivel de la cultura y la sociedad local una serie de elementos propios, como la lengua, la religión, la indumentaria, entre otros muy importantes que definen una identidad. Sin embargo, cuando los procesos de cambio se profundizaron, incidieron de forma dramática en la estructura social, al tiempo que empezaron a gestarse nuevos procesos identitarios.

\section{La sociedad indígena totonaca en el norte de Veracruz}

Con el fin de establecer un marco comparativo haré la caracterización de los pueblos de la región a partir de un caso: Coyutla, municipio de gran importancia por considerarse una de las puertas a la Sierra de Papantla.

Antes de la construcción de la carretera, en la década de los ochenta, Coyutla era un poblado donde predominaban las casas de madera y piedra, así como las chozas de varas y "tarros" (una especie de bambú silvestre). Los techos eran de teja a dos aguas y en algunos casos de cartón o de palma. Los pisos eran de tierra y las viviendas contaban con una o dos piezas que empleaban para múltiples actividades. En su mayoría, las casas no poseían letrinas y se practicaba el fecalismo al aire libre. Había pocos centros de esparcimiento. Los hombres acudían a lugares públicos, como las cantinas, donde se bebía y se jugaba a las cartas o al cubilete. Las mujeres compartían algunas labores como el lavado de ropa en el río o se visitaban en sus hogares para mantenerse comunicadas. Predominaba el monolingüismo en idioma totonaco, sobre todo en las mujeres. La indumentaria de los hombres era el calzón, la camisa de manta, el sombrero, el morral y a veces los huaraches. Las mujeres vestían el quexquemitl (prenda de origen prehispánico), la faja y la nagua (falda indígena).

Cuando se realizaba un matrimonio, éste se asentaba en la casa paterna. Posteriormente, al llegar la descendencia, se acostumbraba levantar una casa en el terreno familiar y se constituía un vecindario de parientes. En esta estructura de familia extensa la primacía la tenía el hombre de mayor edad que podía ser el padre, el abuelo o el hermano mayor. De allí, la autoridad se concedía a las mujeres, quienes asumían el mando del hogar sólo en el caso de la ausencia de varones.

Los juegos infantiles tenían el propósito de preparar a los nuevos miembros de las familias para conocer, adaptarse al medio y perfeccionar las técnicas de subsistencia tanto en los hombres como en las mujeres. El espacio para la recreación entendida como tiempo libre todavía no aparece. Hará falta la concurrencia de ciertos detonantes (centros educativos, caminos, medios tecnológicos) que abrirán una brecha en la trayectoria vital. Así el niño ( $k a^{\prime} h u a^{\prime} z a$ o ka'hua'chu') será, después del matrimonio, un señor (chi'xcú). La niña (tzu'ma'jat), de igual modo, devendrá en señora (puscat), con los derechos y obligaciones que la sociedad indígena otorga. 
En gran medida la estabilidad y permanencia de las instituciones indígenas estaban relacionadas con el matrimonio y la familia. La gran mayoría de sus principios y rituales daban soporte a las relaciones sociales sobre la base de una economía agrícola y creencias religiosas sincréticas. A la vez que se veneraba a los santos se vigilaban los cultivos con técnicas y calendarios plenamente mesoamericanos. En gran medida, el relativo aislamiento de la sociedad nacional permitió que formas ancestrales de organización no sufrieran mayores cambios hasta ya muy avanzado el siglo veinte.

Por lo general, los totonacos seguían sus trayectorias vitales de acuerdo a los ritos de paso establecidos por la propia cultura. De tal suerte que el tránsito de la infancia a la edad adulta se daba entre los 13 y los 15 años aproximadamente. Esto era determinado por los padres quienes concertaban el matrimonio. Los sujetos no intervenían en las decisiones que tomaban en su nombre. Las libertades individuales que gozaban los ciudadanos mexicanos se desconocían o simplemente no se acataban. La sociedad totonaca seguía un curso trazado desde la instauración del orden colonial donde las reformas emanadas por las constituciones liberales poco impactaban en la vida social.

\section{El surgimiento de lo juvenil indígena en El Totonacapan}

Aunque se anunció su construcción desde mayo de 1969, la carretera que une esta parte de la sierra con las ciudades Papantla y Poza Rica no se concluyó sino hasta 1985 (García, 2009). A partir de entonces, esta vía de comunicación se ha constituido como el referente de los grandes cambios en la historia reciente de la región, como un detonante del progreso, al mismo tiempo que el causante del deterioro social de acuerdo a la interpretación de los informantes. Dicho progreso se percibe como el responsable de la aparición de conductas desconocidas en la región como el pandillerismo y la drogadicción de los jóvenes mestizos e indígenas. En voz de un profesor de telesecundaria, originario de Coyutla y no hablante de la lengua indígena, los cambios que se han experimentado han sido dramáticos:

Definitivamente se ha ido perdiendo el amor a nuestras tradiciones porque desafortunadamente nuestras autoridades se han encaminado más a las obra material, a construir caminos, carreteras, escuelas; se dedican mucho a la construcción material, pero no se dedican a la labor social, entonces ya no le dan impulso a la conservación de nuestras tradiciones, lógicamente la feria patronal ya no tiene el mismo impulso, el amor a lo que es la cultura madre, la cultura indígena se ha ido perdiendo porque, precisamente han ido emigrando y, jóvenes que van a lugares como México vienen con otras costumbres y ya sus padres, pues se van olvidando de conservar las tradiciones. (J.J. P. M., 2001).

En la percepción del mundo adulto, indígena y no indígena, hay un pasado "antes" de los cambios en las vías de comunicación, cuando el pueblo aparece como un espacio socialmente organizado y armonioso en el que la gente se encuentra claramente definida y clasificada según su origen: Unos son los "de calzón”, los totonacos y otros, los "de razón", los mestizos. Este orden se va a ver trastocado con la aparición de hijos de indígenas que han migrado o estudiado y que, implícitamente, van a poner en entredicho tal orden, sobre todo cuestionando el control de los padres sobre los hijos, del hombre sobre la mujer, del mestizo sobre el indígena.

Aparece un reacomodo en la estructura del poder familiar cuando los y las jóvenes empiezan a no obedecer ciertas normas establecidas como los matrimonios "arreglados" entre los padres de los contrayentes. Un caso muy parecido se da en las comunidades hñä hñü, del estado mexicano de Hidalgo, donde la lucha por el reconocimiento tiene que ver con la capacidad de que su 
voz sea escuchada, tanto por las autoridades familiares como por las del pueblo (Cortés Rivera, 2011). Si se quiere, podemos entender que en los pueblos indígenas existe un proceso de construcción de una ciudadanía comunitaria por parte de estas juventudes indígenas emergentes, quienes a través de sus actos interpelan toda una estructura social históricamente afianzada.

Antes de esta etapa de surgimiento de nuevas identidades en el escenario local, aparece lo que los mestizos llaman el "indio revestido", el hijo de la "gente de calzón" que no usa la indumentaria de sus padres. Esta categoría es de uso muy común en esta parte de la sierra y es un ejemplo de cómo la sociedad indígena poscolonial conserva muy arraigada la idea de una "indianidad" fijada por tres elementos, a saber: el fenotipo (aspecto físico), la lengua, y la indumentaria. De estos tres elementos se destaca aquel que es más superficial y que por generaciones ha identificado al grupo social, el calzón de manta y la camisa holgada en los hombres y el quexquemitl bordado en las mujeres. Como segmento social oprimido cobra cierta validez la idea, no cabalmente expresada entre algunos los habitantes de la región, de que "hay que desindianizarse para progresar". Un primer elemento para este ascenso en la sociedad local es el abandono de la indumentaria.

Esto llega a percibirse como una negación de "sí mismo". El "indio revestido" aparece como un totonaco "renegado" entre la comunidad no indígena. Inicialmente surge como una figura que subvierte y cuestiona el orden social y abandona temporal o permanentemente estos elementos culturales, supuestamente inamovibles. Al tiempo que hombres y mujeres cambian de indumentaria se les solicita un mejor dominio del español. Es frecuente la expresión: "Si ya se cambió de ropa, es porque ya habla castilla" o "porque ya estudió".

Para muchos mestizos el "indio revestido" es un ser incompleto que no ha logrado dejar de ser "indio" pero que tampoco es un mestizo, ni es aceptado como tal en el esquema de la sociedad local. Se concibe este cambio como una estrategia fallida para ocultar su "indianidad", ya que ellos perciben "lo indio" como una integridad de lengua, aspecto físico e indumentaria.

Los jóvenes indígenas en sentido estricto no son "indios revestidos" debido a que algunos ya no usan la indumentaria tradicional, por lo tanto no se trató de un acto de "revestimiento", pero sobre todo porque tienen a su favor un recurso que otras generaciones no detentaron: un capital cultural ${ }^{4}$ que les posibilita igualarse al mestizo, un capital cultural que obtuvieron a través de la escuela y los medios de comunicación.

Al igual que el "indio revestido", los jóvenes indígenas no usan la indumentaria tradicional, pero tienen a su favor un mejor dominio del español, un nivel educativo que puede llegar a la secundaria o la preparatoria y que les facilita migrar a la ciudad de México. Estas experiencias les permiten forjar un nuevo tipo de conciencia sobre sí mismos y sobre su relación con la sociedad nacional, donde la transformación de costumbres y hábitos de vida no pasa necesariamente por la negación de su legado indígena. ${ }^{5}$ Aunque este fenómeno no es generalizado es posible encontrar una incipiente intelectualidad indígena que se ha formado en recintos educativos y cuya opinión enriquece las diferentes concepciones de la identidad juvenil. ${ }^{6}$ En el polo opuesto se encuentran aquellos que experimentan la migración con una carencia de recursos culturales y económicos que les orilla a ocultar su condición étnica para no ser víctimas de los robos y agresiones que se prodigan en el entorno urbano.

En resumen, son diversos factores (la educación, el trabajo, los medios de comunicación, la migración, etcétera) los que, en su conjunto, proporcionan a los y las jóvenes indígenas elementos para moverse en el mundo contemporáneo de una manera distinta a la generación de sus padres. En un momento dado, saber leer y escribir, emplear computadoras, usar internet o telefonía celular, aspirar a una carrera universitaria, el deseo de descubrir la inmensidad del mundo no 
a través de relatos sino de vivencias propias, altera necesariamente la visión tradicional en la que fueron elaboradas todas sus creencias y costumbres.

\section{Jóvenes mujeres indígenas}

En la sociedad indígena totonaca, antes de los dramáticos cambios ya citados, las mujeres asumían pasivamente el lugar que la comunidad les había asignado. Los padres decidían con quién y cuándo casarse. La virginidad era valor supremo y en la familia el hombre asumía el papel directivo. Las mujeres ayudaban desempeñando labores domésticas que consistían en la cría de animales, la limpieza y cuidado de la casa, el lavado de ropa, la elaboración de alimentos y la crianza de los hijos. La jornada de trabajo iniciaba de madrugada y tenía como centro de gravedad las responsabilidades del hogar, el trabajo en la cocina junto al brasero. Antes del amanecer ellas ya tenían un programa razonado de las actividades prioritarias y se hacían preparativos para el resto del día, el cuidado de los niños y los animales, la limpieza del patio y de la huerta familiar, mientras los hombres se dirigían a la labranza.

El tiempo se regía por los ciclos productivos y sociales. La vida pública era estrictamente regulada a través de un sistema social muy afianzado en la tradición, los grandes eventos sociales eran las bodas, los funerales, las fiestas patronales. El contacto con la sociedad nacional era escaso y casi siempre se consideraba que viajar era tarea de hombres. Las mujeres habitaban un mundo dominado por el monolingüismo, donde el contacto directo con la sociedad nacional era escaso y se daba en términos generalmente administrativos. Estas experiencias no eran tan particulares que cambiaran el curso de las vidas de las mujeres indígenas, ni a raíz de ellas, las mujeres aparecían como interlocutoras ante la sociedad exterior.

En la actualidad parte de ese mundo así como los elementos que le daban firmeza se han transformado y los roles asignados han perdido su eficacia. La escuela es uno de los principales factores en la generación de la capacidad de elegir por parte de las mujeres.

En Coyutla, la escuela es vista como un medio por el cual se busca el ascenso social a través de un trabajo mejor pagado que el de la labor agrícola. No deja de llamar la atención la gran cantidad de hijos e hijas de campesinos que asisten a la escuela secundaria y preparatoria con la esperanza de elevar su calidad y nivel de vida. Por citar un ejemplo, en el caso de dos hermanas indígenas, si una estudia y la otra no, se considera que esta última debe llevar el traje tradicional en virtud de que no es estudiante ni conoce las cosas del mestizo. Igual criterio se aplica para los hombres que cambian el "calzón" por el pantalón. Además se considera condición indispensable dominar "el castilla" para desenvolverse con soltura en el mundo no indígena.

Como antecedente del impacto del factor educativo en los procesos identitarios, quisiera mencionar que un primer momento para rastrear la aparición de la juventud en Coyutla, y por extensión en el totonacapan veracruzano, puede establecerse a partir de la fundación de la primera escuela telesecundaria en 1972, seguida de la secundaria técnica en 1976 y de una escuela de Bachilleres en 1980. Con la fundación de estas escuelas se inicia un proceso de institucionalización de la juventud como una etapa de la vida que no estaba contemplada en la sociedad tradicional. Como ya se mencionó, el tránsito de la infancia a la vida adulta estaba claramente señalado por el matrimonio concertado por los padres de los contrayentes y realizado a una edad muy temprana.

En fechas relativamente recientes (año 2012), se han puesto en marcha los servicios educativos de varios institutos y centros de enseñanza superior, particularmente en Entabladero (Espinal), Coyutla y Zozocolco, que son en su mayoría extensiones de institutos tecnológicos que tienen su sede en Poza Rica, Veracruz. Por sus rasgos muy particulares es necesario destacar el papel de la Universidad Veracruzana Intercultural, una institución académica que tiene como propósito fortalecer las regiones interculturales 
de Veracruz, ${ }^{7}$ fin para el que puso en marcha sus primeras licenciaturas en el año 2005 en el municipio de Espinal.

En este contexto, es de indudable importancia el testimonio que las mujeres estudiantes ofrecen y sobre todo a quién interpelan. En este caso es a la sociedad tradicional y patriarcal de la región. Esta posibilidad de decisión, aunque mínima de acuerdo a los estándares globales, es un elemento significativo del cambio en la sociedad rural e indígena y su relación con las mujeres.

La primera, es una estudiante de secundaria nativa de Coyutla y hablante de totonaco cuyas ideas entorno a la costumbre del matrimonio tradicional son las siguientes:

Mi mamá se casó con la costumbre de antes. [Entonces] A la edad de 9 o 10 años iban a tu casa y hablaban con tus papás y te piden en matrimonio sin conocerte. Tus padres piensan que eso está bien y dicen que sí y te llevan. Entonces ni siquiera haces nada, nada más te casan y es como vender algo. Antes era así, ni siquiera te fijabas en alguien, no importaba si no te gustaba o no te caía bien. Ahora ya no es así, ahora cada quien tiene su novio y antes era distinto... Yo pienso que esa costumbre estaba mal, era como vender un puerco que ni siquiera conoce donde va. Yo no aceptaría casarme de esa forma. Me mataba antes que ir con él o me iría de la casa (A. A. L. 2001).

A continuación, una reflexión de una universitaria que no es hablante de la lengua indígena originaria de Espinal, Veracruz, sobre los cambios culturales y el papel de la mujer en la región:

Con la modernidad y los cambios se ganó una forma de libertad para las mujeres para que tuvieran derecho a la educación, a la libertad, a la forma de pensar y de vestir. Derecho a la toma de decisiones dentro del hogar, ya que antiguamente las mujeres no tenían ni voz ni voto en todo el sentido de la expresión. Las mujeres eran sólo acompañantes del hombre. Ahora no, porque las mujeres tiene el derecho de opinar (B. X. G. C. 2009).

En mi opinión, estas expresiones manifiestan un espíritu de cambio que antes de las transformaciones ya citadas no eran concebibles. La sociedad indígena y rural se mueve a la velocidad de los elementos más sujetos en su estructura que, en este caso, son las mujeres, y las mujeres, aunque lentamente, están cambiando.

\section{Juventud indígena y tiempo libre}

Los cambios que transformaron el orden económico y social también dieron pie al surgimiento de una práctica desconocida: el tiempo libre. Aunque es bien conocido el nacimiento del tiempo libre en la sociedad industrial, poco se ha dicho sobre el nacimiento del tiempo libre en una sociedad rural como la que abordamos.

En el caso de los y las jóvenes totonacos de Coyutla, este tiempo de recreación es de aparición reciente y está ligado a las nuevas obligaciones, deberes y labores que desarrollan. Este tiempo generalmente se invierte en disfrutar de la televisión, la radio, la música, el deporte. Una división primaria del tiempo libre nos sugiere que se han abierto, por lo menos, dos opciones para disfrutar del mismo: un tiempo libre reservado para los espacios públicos y un tiempo libre reservado a los espacios privados, tal como se expresa en el cuadro 1.

Los espacios vivenciales que se han abierto a partir de una reestructuración del aparato productivo, de las escuelas y de las vías de comunicación dificultan una estimación a cerca de cuántas horas diarias se dedican al tiempo libre.

A pesar de lo anterior, es posible identificar los espacios de socialización donde el actor "juvenil" aparece aún en estado germinal. Si en la época anterior a la carretera, la infancia era un periodo de espera donde la boda y el compromiso se sabían inevitables, si los espacios vitales transcurrían entre la milpa y la casa, la 
plaza y la Iglesia, cuatro décadas después esta situación ha cambiado radicalmente. El esparcimiento a través del baile y la cultura física a través del deporte, han hecho su aparición en lo que he denominado "tiempo libre público".

Los usos del tiempo libre también podrían marcar una diferencia entre mujeres y hombres, ya que las mujeres se encuentran sujetas en mayor grado a la vigilancia familiar y por lo tanto el uso del tiempo libre transcurre en el ámbito doméstico y fuera de él con el círculo de amistades femeninas. Por el contrario, a los hombres se les permite mayor libertad en el uso del tiempo libre con los amigos que a las mujeres. Esto tal vez pueda explicar la percepción de que entre las jóvenes existen lazos más profundos, leales y duraderos que entre los hombres, aparte de que estos últimos presentan, en algunos casos, cuadros de adicción a sustancias y conductas pandilleriles, situación desconocida entre las mujeres.

Eldeporte es una práctica pública mayoritariamente masculina y las disciplinas más populares son el futbol, organizado en una liga local, y el basquetbol. También existen eventos que congregan a ambos sexos. En el auditorio de la "Asociación Ganadera Local" se realizan fiestas con música viva y "sonido disco" (mediante un equipo de alta fidelidad operado por una o dos personas). Las fiestas estudiantiles tienen pues dos espacios: uno propio, las instalaciones escolares y uno temporal, el auditorio. En la opinión de un joven empleado municipal nativo no hablante de totonaco, tal fenómeno es de aparición reciente:

Las fiestas se dan entre los jóvenes de las preparatorias y telesecundarias. Empezaron hace como 10 años. Son un poquito diferentes a las de antes cuando no había "sonido". A los chavos [hombres y mujeres] les gusta la música y bailan. Cuando un joven quiere sacar a bailar a una muchacha le pide permiso a ella directamente. A esas fiestas casi no van las muchachas de nahua, de vestido tradicional, y si van casi no bailan (I. B. 2000).
¿Qué escucha cada grupo social? En voz de una joven estudiante de preparatoria que no es hablante del totonaco el tema puede verse de la siguiente manera:

Entre los jóvenes hay una distinción de gustos musicales. La gente de calzón prefiere la música de banda; en cambio, la gente de "vestido", la música pop (M. A. M. 2001).

Desde mi punto de vista esta división no es tajante, sin embargo, revela que en la conformación de los gustos se continúa percibiendo a la sociedad dividida en grupos sociales claramente separados. En esta distinción, por ejemplo, no tienen cabida los jóvenes migrantes y los estudiantes que gustan del rock, el hip hop y el pop. También es necesario anotar que la música es un evento de goce privado, merced a la existencia de aparatos reproductores que antes no existían y es una afición, bien sea para escuchas o ejecutantes. Una joven estudiante de secundaria, cuyos padres son campesinos y usan la indumentaria tradicional de la región, comparte:
Al llegar de la escuela lo primero que hago es revisar todas mis materias y luego me voy a comer y después veo como media hora de televisión. Después voy a la biblioteca a estudiar o lo que sea pero casi no me dejan salir. Le ayudo a mi mamá a hacer el quehacer cuando estoy en la casa (A.A. L. 2001).

Para un joven estudiante de preparatoria, hablante de totonaco, la música representa una diversidad de temas:

Cuando tengo tiempo libre lo dedico a la música. Por ejemplo, con unos cuates estoy aprendiendo a tocar el violín y guitarra. Mi intención no es aprender huapangos sino crearle un nuevo sonido al violín... Me crie en la música, mi papá sabe el son y me lo enseñó. Pero en lo personal no me motiva el huapango. La música que me gusta está apegada a la rondalla. 
También me gusta Arjona. Me gusta la música que te puede cambiar el estado de ánimo. Hay música que te relaja y otra que te pone de mal humor. No me gustan las canciones rancheras ni el rock porque invitan a los jóvenes a "dispersionarse", ni Vicente Fernández porque se expresa mal de las mujeres y como que invitan a tomar. Yo no tomo ni fumo (M. P. G. 2000).

De acuerdo con un estudiante y campesino totonaco, las preferencias estéticas son objeto de una cuidadosa elección:

A mí me gusta el rock, la música norteña desde chico por estar viendo la tele y la radio. Me gusta el sonido que es diferente de los norteños. Así empecé a desarrollar esos gustos. Las canciones del Tri no me gustan mucho, solamente las de Maná, Caifanes, y del rock fuera de México, pues Bon Jovi, Scorpions, Metallica (J. G. G. 2000).

Otra afición destacable por su novedad es la lectura. Enteramente relacionada con la aparición de la escuela y la creación de aspiraciones nuevas entre los jóvenes estudiantes, merece un tiempo que algunos le dedican. Para una estudiante de preparatoria hablante del totonaco, el disfrute de la música se puede combinar con el goce de la lectura:

Dentro de mi vida normal, en mi tiempo libre, leo sobre la adolescencia, novelas sobre el matrimonio ya que a mí me gustaría casarme como a los 26 años. En el tiempo en que no tengo nada que hacer me pongo a escuchar música romántica, cumbias, pero me gusta más la romántica. También me gusta escuchar poemas, el poema del "Cristo roto", también veo televisión, me gustan las novelas (M. P. S. 2001).

Es evidente que la existencia de aparatos receptores de radio y televisión han modificado los hábitos de la sociedad en El Totonacapan. A diferencia de hace 40 años, estos aparatos son ahora más accesibles, siendo las computadoras y la conexión a internet los nuevos instrumentos de estudio, trabajo y esparcimiento de las nuevas generaciones. No obstante su posesión y uso, aunque en ascenso, es todavía limitado. Los recursos tecnológicos más comunes son las televisiones y reproductores de discos compactos y, a través de ellos, consumen la oferta cultural de los medios. Como testimonia una estudiante de secundaria hablante de totonaco:
A mí me gusta todo tipo de música, el rock, la música pop, Christina Aguilera, Thalía, Paulina Rubio. El huapango no me gusta porque no lo sé bailar, aunque no es tan difícil. Cuando tengo tiempo libre veo la tele, programas de concursos y telenovelas. También escucho el radio, me gustan las estaciones "Fiesta Mexicana" y "Stereo Rey" y un programa los domingos que pasa en cadena nacional (G. J. C. 2001).

El balance que hace un joven estudiante indígena sobre la sociedad, los medios y el tiempo libre en Coyutla constituye un ejemplo del pensamiento reflexivo al interior de la sociedad local, ya que asume una postura crítica y poco favorable a los efectos positivos de los medios de comunicación:

A veces los hijos se pelean con los padres, la causa de esto son los medios de comunicación, la era digital, todo forma parte de eso. He analizado las cosas, si no hubiera esos programas como "Derbez en Cuando", que utilizan un vocabulario desgraciadamente vulgar y pasan a traer a toda la gente. Por eso ya hay libertad de decir cualquier cosa. Nos afecta que no se respete a los padres.iQué pasa con la música del "Tri""? Ya todo lo toman a juego y no es cierto. Lo principal en la vida no es decir tonterías, sino compartir las cosas, vivir la vida en armonía. Hay muchas cosas malas que presentan los medios de comunicación. Yo voy formando mi propia opinión pero también tengo que 
respetar el pensamiento de los demás. Quiero a mi familia pero no tengo que estar siempre con ellos. De todos modos hay confianza y respeto (M. P. G 2000).

Así es como, a partir del relato de los propios actores, podemos ir articulando una visión panorámica sobre los elementos que integran esta nueva identidad juvenil indígena, que tiene como su referente inmediato la interacción con la sociedad local y el conocimiento de modelos de sociabilidad propios de los ámbitos nacionales e internacionales, estos últimos, a través del acceso a las nuevas tecnologías de la información y la comunicación.

\section{Conclusiones}

La juventud en el Totonacapan veracruzano es un fenómeno de reciente aparición, consecuencia de los procesos de cambio que dinamizaron el contacto de los grupos indígenas con la sociedad nacional. Esto repercutió en la aparición de nuevas identidades, que reconozco bajo la etiqueta genérica de juventud indígena, aunque tal vez fuera más apropiado usar el término en plural, por las diversas maneras en que esta juventud se manifiesta. Estas juventudes coexisten con los valores tradicionales de sus padres y abuelos, y al mismo tiempo, han desarrollado nuevas formas de socialización a través de la educación, el trabajo, la política, la religión y el uso del tiempo libre. Son jóvenes que piensan y hacen cosas que no estaban contempladas en la vida tradicional, aspectos tales como la música, el deporte y el uso de medios de entretenimiento electrónico como la radio, internet o la telefonía celular.

$\mathrm{Al}$ alterarse el estilo de vida campesino, los límites simbólicos de las etapas de la vida agraria también sufrieron cambios. Si en la vida del totonaco "tradicional" eran vitales las ceremonias del nacimiento, el bautismo, el matrimonio o la muerte, que de alguna manera marcaban o señalaban las etapas de la vida, este mismo ordenamiento ha sufrido alteraciones y propiciado la aparición, entre la niñez y la edad adulta, de un periodo novedoso y reconocible como el de la juventud. Este periodo de juventud indígena coincide con los tiempos designados anteriormente para el compromiso del matrimonio concertado por los padres, aproximadamente a la edad de doce o trece años, mientras que ahora corresponde a la etapa en que ellos mismos ingresan a la escuela secundaria para continuar sus estudios. Aquellos que no siguen estudiando examinan la posibilidad de emigrar a la ciudad de México, la frontera o los Estados Unidos en la búsqueda de un trabajo mejor remunerado.

Dicho periodo de juventud, considero, tiene una duración variable que termina con el matrimonio. El matrimonio es la señal más clara de que los jóvenes han dejado de serlo y se incorporan plenamente al mundo adulto, a la cultura parental, con todos sus derechos y obligaciones. No obstante, aún sin llegar a la adultez reconocida, la juventud indígena puede aspirar a un cargo religioso, a la propiedad de las tierras, al liderazgo de un negocio propio, al ejercicio de una profesión o la práctica de nuevos oficios.

La aparición del estatus de joven y la construcción de la identidad juvenil no son fenómenos acabados sino en proceso. La sociedad local, en ciertos aspectos, no termina de asimilar el papel de sus nuevos integrantes. Existe temor por parte de ciertos sectores conservadores a que estos cambios signifiquen el fin de la cultura indígena. En nuestra opinión, estas transformaciones implican el principio de nuevas relaciones de los pueblos originarios con las sociedades nacionales y globales, en las que para tener un mayor margen de negociación se aprende del otro, buscando mantener las propias raíces. O como dijo un joven miembro de esta generación de totonacos: "tomar lo de ellos y ser yo mismo". En razón de que los pueblos indígenas han formulado múltiples estrategias de sobrevivencia, una de ellas puede ser la aparición de nuevas identidades como las juventudes que cambian el sentido de las trayectorias de vida para adaptarse a un mundo cambiante y no desaparecer. 


\section{Notas}

${ }^{1}$ Los datos etnográficos presentados en este artículo fueron registrados durante el trabajo de campo realizado los años 2001, 2009 y 2012. Las dos primeras fechas corresponden a la investigación para mi tesis de maestría en antropología social en el Centro de Investigaciones y Estudios Superiores en Antropología Social (CIESAS), titulada "juventud indígena en Coyutla: construcción de identidades en el espacio rural", que fue posible gracias a una beca otorgada por el Consejo Nacional de Ciencia y Tecnología (CONACYT) para el periodo 1999-2001. La temporada de campo del año 2012, se realizó en el marco del proyecto "Identidad y educación en las juventudes indígenas y rurales de Coyutla, Veracruz", con financiamiento del Instituto de Veracruzano de la Cultura (IVEC) y el Consejo Nacional para la Cultura y las Artes (CNCA).

2 Es decir, la sociedad que se concibe a sí misma como mestiza en el resto del país.

${ }^{3}$ Escritura de acuerdo al Diccionario totonaco de Xicotepec de Juárez, Puebla, de Ruth Bishop y Aileen Reid (1974).

${ }^{4}$ En el sentido que le otorga Pierre Bourdieu (1987), al referirse a los estados incorporado e institucionalizado del capital cultural.

${ }^{5}$ Aunque este estudio no se centra especialmente en el tema de la migración, la sugerencia de mis lectores me permitió encontrar paralelismos con los hallazgos de Marielle Pepin Lehalleur sobre los indígenas mixtecos quienes, para evadir la discriminación, ocultan los signos "más evidentes" de la indianidad, como la vestimenta y la lengua, al incorporar las competencias y atributos de los mestizos, poniendo en marcha una especie de "mestizaje estratégico" sin por ello dejar de amar su cultura y orígenes (Pepin Lehalleur, 2006: 110$111)$.

${ }^{6}$ Es notoria la presencia de estudiantes indígenas que han llegado a cursar carreras profesionales en universidades públicas y privadas en el periodo que abarca las últimas cuatro décadas, intensificándose en fechas recientes por la aparición de la Universidad Veracruzana Intercultural.

${ }^{7}$ Algunos de los objetivos de la Universidad Veracruzana Intercultural son: I. Promover el desarrollo de programas de educación superior que contribuyan al fortalecimiento de las regiones interculturales del estado de Veracruz, es decir, de aquellas regiones en las que confluyen, por razones históricas, grupos humanos que pertenecen a culturas descendientes de poblaciones indígenas originarias de lo que hoy es el territorio veracruzano, poblaciones indígenas provenientes de otros estados de la república, grupos y personas que aunque vienen de otros países también han decidido vivir en el mismo territorio, así como poblaciones mestizas asentadas en esas regiones. II. Formar profesionales capaces de responder a las demandas de la sociedad en sus regiones de origen y del estado en general, con programas académicos transdisciplinares desde la perspectiva intercultural, en http://www. uv.mx/uvi/universidad/identidad.html [consultado: 20 de agosto de 2009].

${ }^{8}$ Programa de comedia mexicana, muy popular en los primeros años del siglo XXI.

9 Grupo de rock mexicano, emblemático para las juventudes rockeras mexicanas desde la década de los ochenta del siglo pasado.

${ }^{10}$ http://www.lavida.org.mx/media-gallery/detail/50l/896 [consultado: 10 de junio de 2012].

\section{Bibliografía}

Bishop, Ruth G. y Aileen A. Reid (1974), Diccionario totonaco de Xicotepec de Juárez, Puebla: Totonaco-castellano, castellano-totonaco, México, Instituto Lingüístico de Verano. Bourdieu, Pierre (1987), "Los tres estados del capital cultural" en Sociológica, Revista del Departamento de Sociología, año 2, núm. 5, UAM-Azcapotzalco, en http://www.revistasociologica.com.mx/pdf/0503. pdf, [consultado: 18 de abril de 2012]. 
Cortés Rivera, Dalia (2011), “Las juventudes hñahñu en contextos de migración: los que se quedan", en La jornada del campo, Suplemento Informativo de La Jornada núm. 45, México, en http://www.jornada. unam.mx/2011/06/18/juventud.html, [consultado: 5 de julio de 2011].

Chenaut, Victoria (1995), Aquellos que vuelan. Los totonacos en el siglo XIX, México, CIESAS-INI.

García Martínez, Ariel (2009), Juventud indígena en Coyutla: construcción de identidades en el espacio rural. Tesis para optar el título de Maestro en Antropología Social, México, CIESAS.

Pepin Lehalleur, Marielle (2006), "Des réseaux aux communautés de pratiques. Ethnographie de trois vagues migratoires depuis la côte du Oaxaca (Mexique)"In Cahiers des Amériques latines, núm. 51-52, Paris:Institut des hautes études de l'Amérique latine, en http://www.iheal.univ-paris3.fr/IMG/CAL/cal5l52-dossier7.pdf, [consultado: 4 de junio de 2012].

Pérez Ruiz, Maya Lorena, Coord. (2008), Jóvenes Indígenas y globalización en América Latina, México: INAH.

Reguillo, Rossana Coord. (2010), Los jóvenes en México, México: Fondo de Cultura Económica, Consejo Nacional para la Cultura y las Artes.
Urteaga Castro-Pozo, Maritza (2010), “Género, clase y etnia. Los modos de ser joven" en Reguillo, Rossana (Coord.), Los jóvenes en México, México: Fondo de Cultura, pp. 15-5l.

Urteaga Castro-Pozo, Maritza (2008), "Lo juvenil en lo étnico. Migración juvenil indígena en la sociedad contemporánea mexicana" en Ponto-e-Vírgula, núm.4, São Paulo, revista do Programa de Estudos PósGraduados em Ciências Sociais da PUC-SP, en http:// www.pucsp.br/ponto-e-virgula/n4/dossie/pdf/ ART9MaritzaUCPozo.pdf, [consultado: 21 de mayo de 2012].

Valenzuela Arce, José Manuel (2009), El futuro ya fue. Socioantropología de l@s jóvenes en la modernidad, México: Colegio de la Frontera Norte.

Valenzuela Arce, José Manuel (1997), “Culturas juveniles. Identidades transitorias”, en Revista JOVENes. Revista de estudios sobre juventud, añol, núm. 3, enero-marzo, cuarta época, pp.12-35.

Velázquez, Emilia (1996), "Mercados y Tianguis en el Totonacapán Veracruzano", en Chenaut, Victoria (coord.), Procesos rurales e historia regional: (sierras y costas totonacas de Veracruz), México: CIESAS, pp. 157181. 
Cuadro 1. Tiempo Libre en el Espacio Rural

\begin{tabular}{|c|c|c|}
\hline & $\begin{array}{c}\text { Tiempo libre en espacios } \\
\text { públicos }\end{array}$ & $\begin{array}{c}\text { Tiempo libre en espacios } \\
\text { privados }\end{array}$ \\
\hline Lugares & $\begin{array}{l}\text { - Auditorio de la asociación } \\
\text { ganadera local } \\
\text { - El parque } \\
\text { - Las canchas deportivas } \\
\text { - Las calles }\end{array}$ & - La casa familiar \\
\hline Actividades relacionadas & $\begin{array}{l}\text { - } \text { Bailes populares y } \\
\text { escolares } \\
\text { - Fiestas de quince años } \\
\text { - Reuniones informales } \\
\text { - Cortejo } \\
\text { - Actividades deportivas }\end{array}$ & $\begin{array}{l}\text { - Uso de la radio y la } \\
\text { televisión } \\
\text { - Uso de aparatos } \\
\text { reproductores de música } \\
\text { - Lectura recreativa }\end{array}$ \\
\hline
\end{tabular}

Fuente: elaboración propia.

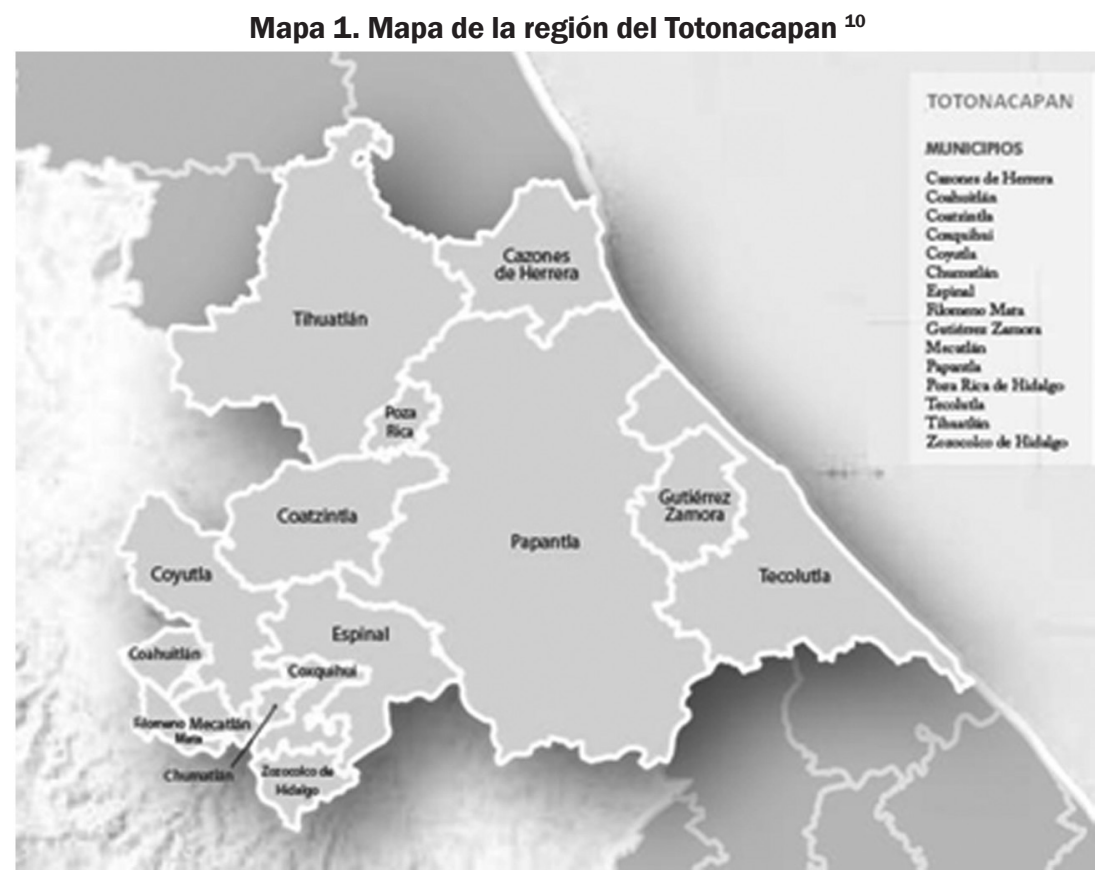

Fuente: http://www.lavida.org.mx/media-gallery/detail/501/896, [consultado: 10 de junio de 2012]. 